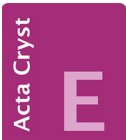
COMMUNICATIONS

ISSN 2056-9890

\section{Crystal structure of 8-bromo-4-oxo-4H- chromene-3-carbaldehyde}

\section{Yoshinobu Ishikawa}

School of Pharmaceutical Sciences, University of Shizuoka, 52-1 Yada, Suruga-ku, Shizuoka 422-8526, Japan. *Correspondence e-mail: ishi206@u-shizuoka-ken.ac.jp

Received 9 July 2015; accepted 10 July 2015

Edited by M. Zeller, Youngstown State University, USA

In the title compound, $\mathrm{C}_{10} \mathrm{H}_{5} \mathrm{BrO}_{3}$, a brominated 3-formylchromone, all atoms are essentially coplanar (r.m.s. $=0.0104 \AA$ for the non-H atoms), with the largest deviation from the least-squares plane $[0.028(5) \AA]$ being for one of the benzene $\mathrm{C}$ atoms. In the crystal, molecules are linked through $\mathrm{C}-$ $\mathrm{H}$... O hydrogen bonds, which are further assembled by faceto-face $\pi-\pi$ stacking interactions [centroid-centroid distance between the pyran rings $=3.854$ (4) $\AA$ ]. Shorter contacts than the sum of van der Waals radii are observed between the $\mathrm{Br}$ and formyl $\mathrm{O}$ atoms $[\mathrm{Br} \cdots \mathrm{O}=3.046$ (4) $\AA, \mathrm{C}-\mathrm{Br} \cdots \mathrm{O}=$ $175.23(18)^{\circ}$ and $\mathrm{Br} \cdots \mathrm{O}-\mathrm{C}=132.6(3)^{\circ}$, features that do indicate halogen bonding.

Keywords: crystal structure; chromone; hydrogen bonding; halogen bonding; $\pi-\pi$ stacking.

CCDC reference: 1412014

\section{Related literature}

For related structures, see: Ishikawa $(2014 a, b)$. For halogen bonding, see: Auffinger et al. (2004); Metrangolo et al. (2005); Wilcken et al. (2013); Sirimulla et al. (2013); Persch et al. (2015); Metrangolo \& Resnati (2014); Mukherjee \& Desiraju (2014).<smiles>O=Cc1coc2c(Br)cccc2c1=O</smiles>

\section{Experimental}

2.1. Crystal data

$$
\mathrm{C}_{10} \mathrm{H}_{5} \mathrm{BrO}_{3} \quad M_{r}=253.05
$$

Monoclinic, $C 2 / c$

$a=27.908(14) \AA$

$b=3.854(3) \AA$

$c=19.145(10) \AA$

$\beta=123.75(4)^{\circ}$

$V=1712.1(18) \AA^{3}$

\subsection{Data collection}

Rigaku AFC-7R diffractometer Absorption correction: $\psi$ scan (North et al., 1968)

$T_{\text {min }}=0.546, T_{\max }=0.715$

2556 measured reflections

1940 independent reflections

\subsection{Refinement}

$R\left[F^{2}>2 \sigma\left(F^{2}\right)\right]=0.037$

$w R\left(F^{2}\right)=0.102$

$S=1.02$

1940 reflections

127 parameters

$\mathrm{H}$-atom parameters constrained

$\Delta \rho_{\max }=1.40 \mathrm{e} \AA^{-3}$

$\Delta \rho_{\min }=-1.27{\mathrm{e} \AA^{-3}}^{-3}$

Table 1

Hydrogen-bond geometry $\left(\AA{ }^{\circ}\right)$.

\begin{tabular}{llllc}
\hline$D-\mathrm{H} \cdots A$ & $D-\mathrm{H}$ & $\mathrm{H} \cdots A$ & $D \cdots A$ & $D-\mathrm{H} \cdots A$ \\
\hline $\mathrm{C} 10-\mathrm{H} 5 \cdots \mathrm{O} 2^{\mathrm{i}}$ & 0.95 & 2.54 & $3.375(5)$ & $147(1)$ \\
$\mathrm{C} 7-\mathrm{Br} 1 \cdots 3^{\text {ii }}$ & $1.89(1)$ & $3.05(1)$ & $4.934(6)$ & $175(1)$ \\
$\mathrm{C} 10-\mathrm{O} 3 \cdots \mathrm{Br}^{\mathrm{iii}}$ & $1.21(1)$ & $3.05(1)$ & $3.962(6)$ & $133(1)$ \\
\hline $\begin{array}{l}\text { Symmetry codes: } \\
-x+2, y+1,-z+\frac{3}{2} .\end{array}$ & & $-x+\frac{3}{2},-y+\frac{3}{2},-z+1 ;$ & (ii) & $-x+2, y-1,-z+\frac{3}{2} ; \quad$ (iii)
\end{tabular}

Data collection: WinAFC Diffractometer Control Software (Rigaku, 1999); cell refinement: WinAFC Diffractometer Control Software; data reduction: WinAFC Diffractometer Control Software; program(s) used to solve structure: SIR92 (Altomare et al., 1994); program(s) used to refine structure: SHELXL97 (Sheldrick, 2008); molecular graphics: CrystalStructure (Rigaku, 2010); software used to prepare material for publication: CrystalStructure.

\section{Acknowledgements}

The University of Shizuoka is acknowledged for instrumental support.

Supporting information for this paper is available from the IUCr electronic archives (Reference: ZL2634).

\section{References}

Altomare, A., Cascarano, G., Giacovazzo, C., Guagliardi, A., Burla, M. C., Polidori, G. \& Camalli, M. (1994). J. Appl. Cryst. 27, 435.

Auffinger, P., Hays, F. A., Westhof, E. \& Ho, P. S. (2004). Proc. Natl Acad. Sci. USA, 101, 16789-16794.

Ishikawa, Y. (2014a). Acta Cryst. E70, o555.

Ishikawa, Y. (2014b). Acta Cryst. E70, o996.

Metrangolo, P., Neukirch, H., Pilati, T. \& Resnati, G. (2005). Acc. Chem. Res. 38, 386-395.

Metrangolo, P. \& Resnati, G. (2014). IUCrJ, 1, 5-7.

Mukherjee, A. \& Desiraju, G. R. (2014). IUCrJ, 1, 49-60.

North, A. C. T., Phillips, D. C. \& Mathews, F. S. (1968). Acta Cryst. A24, $351-$ 359.

Persch, E., Dumele, O. \& Diederich, F. (2015). Angew. Chem. Int. Ed.. 54, 3290-3327. 


\section{data reports}

Rigaku (1999). WinAFC Diffractometer Control Software. Rigaku Corporation, Tokyo, Japan.

Rigaku (2010). CrystalStructure. Rigaku Corporation, Tokyo, Japan.

Sheldrick, G. M. (2008). Acta Cryst. A64, 112-122.
Sirimulla, S., Bailey, J. B., Vegesna, R. \& Narayan, M. (2013). J. Chem. Inf. Model. 53, 2781-2791.

Wilcken, R., Zimmermann, M. O., Lange, A., Joerger, A. C. \& Boeckler, F. M. (2013). J. Med. Chem. 56, 1363-1388. 


\section{supporting information}

Acta Cryst. (2015). E71, o572-0573 [https://doi.org/10.1107/S2056989015013250]

\section{Crystal structure of 8-bromo-4-oxo-4H-chromene-3-carbaldehyde}

\section{Yoshinobu Ishikawa}

\section{S1. Comment}

Halogen bonding has attracted much attention in medicinal chemistry, chemical biology, supramolecular chemistry and crystal engineering (Auffinger et al., 2004, Metrangolo et al., 2005, Wilcken et al., 2013, Sirimulla et al., 2013, Metrangolo \& Resnati, 2014, Mukherjee \& Desiraju, 2014, Persch et al., 2015). I have recently reported the crystal structures of monobrominated 3-formylchromones 6-bromo-4-oxo-4H-chromene-3-carbaldehyde (Ishikawa, 2014a) and 7-bromo-4-oxo-4H-chromene-3-carbaldehyde (Ishikawa, 2014b). Halogen bonding is observed between the formyl oxygen atom and the bromine atom at 6-position in 6-bromo-4-oxo-4H-chromene-3-carbaldehyde (Fig. 1a). On the other hand, a type II halogen $\cdots$ halogen contact (Metrangolo \& Resnati, 2014, Mukherjee \& Desiraju, 2014) is found between the bromine atoms at 7-position in 7-bromo-4-oxo-4H-chromene-3-carbaldehyde (Fig. 1b). As part of my investigation into these types of chemical bonding, I herein report the crystal structure of the monobrominated 3-formylchromone 8bromo-4-oxo-4H-chromene-3-carbaldehyde. The objective of this study is to reveal whether short contacts are observed for the bromine atom at 8-position in the solid state.

The mean deviation of the least-square planes for the non-hydrogen atoms is $0.0104 \AA$, and the largest deviation is 0.028 (5) $\AA$ for the $\mathrm{C} 6$ atom. These mean that these atoms are essentially coplanar (Fig. 2). In the crystal, the molecules are linked through $\mathrm{C}-\mathrm{H} \cdots \mathrm{O}$ hydrogen bonds between the inversion-symmetry equivalents ${ }^{\mathrm{i}}[\mathrm{i}:-x+3 / 2,-y+3 / 2,-z+1 / 2]$, which are further assembled by face-to-face $\pi$ - $\pi$ stacking interactions [centroid-centroid ${ }^{\text {ii }}$ distance between the pyran rings of the $4 H$-chromene units $=3.854$ (4) $\AA$, ii: $x, y+1, z]$, as shown in Fig. 3. Shorter contacts than the sum of van der Waals radii are observed between the bromine atoms at 8-position and the formyl $\mathrm{O}$ atoms $\left[\mathrm{Br} 1 \cdots \mathrm{O} 3^{\mathrm{iii}}=3.046\right.$ (4) $\AA$, $\mathrm{C} 7-$

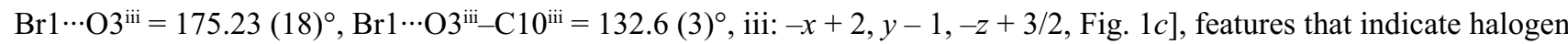
bonding.

\section{S2. Experimental}

To a solution of 3-bromo-2-hydroxyacetophenone $(11.3 \mathrm{mmol})$ in $\mathrm{N}, \mathrm{N}$-dimethylformamide $(20 \mathrm{ml})$ was added dropwise $\mathrm{POCl}_{3}(28.3 \mathrm{mmol})$ at $0{ }^{\circ} \mathrm{C}$. After the mixture was stirred for $15 \mathrm{~h}$ at room temperature, water $(50 \mathrm{ml})$ was added. The precipitates were collected, washed with water and dried in vacuo (yield: $55 \%$ ). $\left.{ }^{1} \mathrm{H} \mathrm{NMR} \mathrm{(400} \mathrm{MHz,} \mathrm{CDCl}_{3}\right): \delta=7.40$ (t, $1 \mathrm{H}, J=7.8 \mathrm{~Hz}$ ), 7.99 (dd, 1H, $J=1.4$ and $7.8 \mathrm{~Hz}$ ), 8.26 (dd, $1 \mathrm{H}, J=1.4$ and $8.3 \mathrm{~Hz}$ ), 8.62 (s, 1H), 10.38 (s, 1H). Single crystals suitable for X-ray diffraction were obtained from a 1,2-dimethoxyethane solution of the title compound at room temperature.

\section{S3. Refinement}

The $\mathrm{C}\left(s p^{2}\right)$-bound hydrogen atoms were placed in geometrical positions $\left[\mathrm{C}-\mathrm{H} 0.95 \AA, U_{\mathrm{iso}}(\mathrm{H})=1.2 U_{\mathrm{eq}}(\mathrm{C})\right]$, and refined using a riding model. 

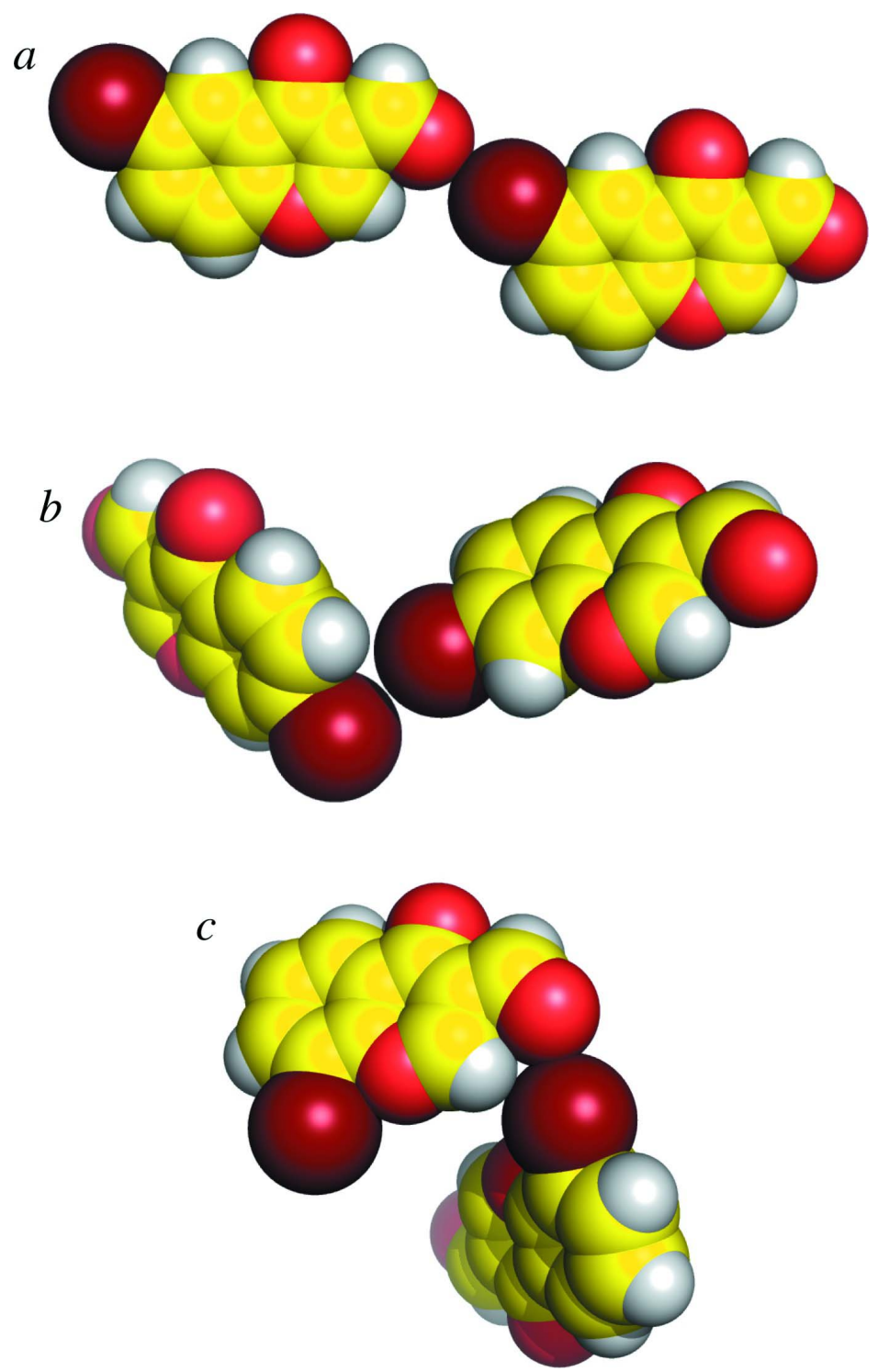

Figure 1

Sphere models of the crystal structures of (a) 6-bromo-4-oxo-4H-chromene-3-carbaldehyde (Ishikawa, 2014a), (b) 7bromo-4-oxo-4H-chromene-3-carbaldehyde (Ishikawa, 2014b) and (c) the title compound (this work). 


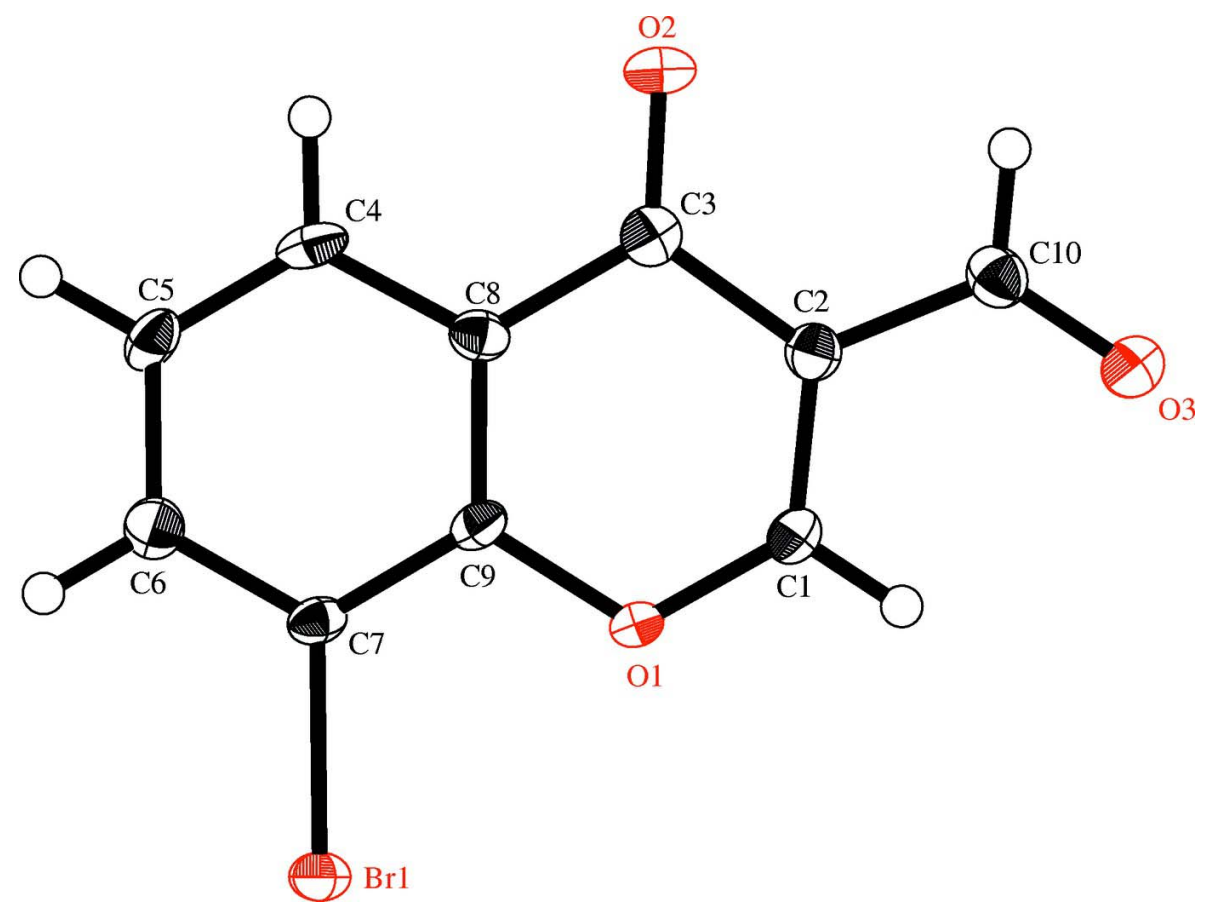

Figure 2

The molecular structure of the title compound with displacement ellipsoids drawn at the $50 \%$ probability level. Hydrogen atoms are shown as small spheres of arbitrary radius.

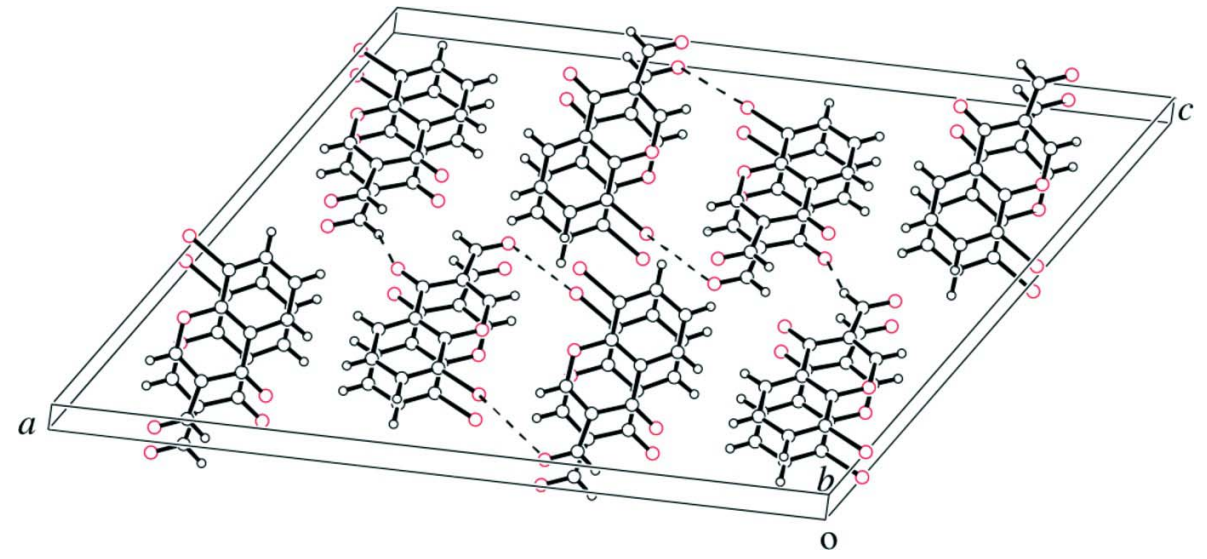

Figure 3

A packing view of the title compound. $\mathrm{C}-\mathrm{H} \cdots \mathrm{O}$ hydrogen bonds and $\mathrm{Br} \cdots \mathrm{O}$ halogen bonds are represented by dashed lines.

\section{8-Bromo-4-oxo-4H-chromene-3-carbaldehyde}

\section{Crystal data}

$\mathrm{C}_{10} \mathrm{H}_{5} \mathrm{BrO}_{3}$

$M_{r}=253.05$

Monoclinic, $C 2 / c$

Hall symbol: -C 2yc

$a=27.908$ (14) $\AA$

$b=3.854$ (3) $\AA$

$$
\begin{aligned}
& c=19.145(10) \AA \\
& \beta=123.75(4)^{\circ} \\
& V=1712.1(18) \AA^{3} \\
& Z=8 \\
& F(000)=992.00 \\
& D_{\mathrm{x}}=1.963 \mathrm{Mg} \mathrm{m}^{-3}
\end{aligned}
$$


Mo $K \alpha$ radiation, $\lambda=0.71069 \AA$

Cell parameters from 25 reflections

$\theta=15.0-17.3^{\circ}$

$\mu=4.79 \mathrm{~mm}^{-1}$

Data collection

Rigaku AFC-7R

diffractometer

$\omega$ scans

Absorption correction: $\psi$ scan

(North et al., 1968)

$T_{\min }=0.546, T_{\max }=0.715$

2556 measured reflections

1940 independent reflections

\section{Refinement}

Refinement on $F^{2}$

$R\left[F^{2}>2 \sigma\left(F^{2}\right)\right]=0.037$

$w R\left(F^{2}\right)=0.102$

$S=1.02$

1940 reflections

127 parameters

0 restraints

Primary atom site location: structure-invariant direct methods
$T=100 \mathrm{~K}$

Plate, yellow

$0.37 \times 0.10 \times 0.07 \mathrm{~mm}$

1280 reflections with $F^{2}>2.0 \sigma\left(F^{2}\right)$

$R_{\text {int }}=0.020$

$\theta_{\text {max }}=27.5^{\circ}$

$h=-20 \rightarrow 36$

$k=-4 \rightarrow 2$

$l=-24 \rightarrow 20$

3 standard reflections every 150 reflections

intensity decay: $-0.8 \%$

Secondary atom site location: difference Fourier map

Hydrogen site location: inferred from neighbouring sites

$\mathrm{H}$-atom parameters constrained

$w=1 /\left[\sigma^{2}\left(F_{\mathrm{o}}{ }^{2}\right)+(0.0578 P)^{2}+0.244 P\right]$

where $P=\left(F_{\mathrm{o}}{ }^{2}+2 F_{\mathrm{c}}{ }^{2}\right) / 3$

$(\Delta / \sigma)_{\max }=0.004$

$\Delta \rho_{\max }=1.40 \mathrm{e} \AA^{-3}$

$\Delta \rho_{\min }=-1.27$ e $\AA^{-3}$

Special details

Refinement. Refinement was performed using all reflections. The weighted $R$-factor $(w R)$ and goodness of fit $(S)$ are based on $F^{2}$. R-factor (gt) are based on $F$. The threshold expression of $F^{2}>2.0 \sigma\left(F^{2}\right)$ is used only for calculating $R$-factor (gt).

Fractional atomic coordinates and isotropic or equivalent isotropic displacement parameters $\left(\AA^{2}\right)$

\begin{tabular}{lllll}
\hline & $x$ & $y$ & $z$ & $U_{\text {iso }} * / U_{\text {eq }}$ \\
\hline Br1 & $1.005108(15)$ & $-0.24742(12)$ & $0.90755(2)$ & $0.01865(14)$ \\
O1 & $0.93556(11)$ & $0.0942(8)$ & $0.73881(17)$ & $0.0180(6)$ \\
O2 & $0.76802(12)$ & $0.4314(9)$ & $0.60186(18)$ & $0.0261(8)$ \\
O3 & $0.86856(12)$ & $0.5554(9)$ & $0.50725(18)$ & $0.0277(8)$ \\
C1 & $0.91204(17)$ & $0.2414(12)$ & $0.6633(3)$ & $0.0188(8)$ \\
C2 & $0.85673(18)$ & $0.3611(12)$ & $0.6140(3)$ & $0.0188(9)$ \\
C3 & $0.81785(19)$ & $0.3273(11)$ & $0.6431(3)$ & $0.0199(10)$ \\
C4 & $0.81361(17)$ & $0.1171(13)$ & $0.7636(3)$ & $0.0220(10)$ \\
C5 & $0.83890(17)$ & $-0.0311(12)$ & $0.8415(3)$ & $0.0201(10)$ \\
C6 & $0.89618(18)$ & $-0.1386(13)$ & $0.8849(3)$ & $0.0212(10)$ \\
C7 & $0.92747(16)$ & $-0.0988(12)$ & $0.8491(3)$ & $0.0161(9)$ \\
C8 & $0.84441(18)$ & $0.1649(11)$ & $0.7261(3)$ & $0.0171(10)$ \\
C9 & $0.90209(16)$ & $0.0568(11)$ & $0.7709(3)$ & $0.0165(9)$ \\
C10 & $0.83814(19)$ & $0.5220(12)$ & $0.5332(3)$ & $0.0216(10)$ \\
H1 & 0.9357 & 0.2646 & 0.6422 & $0.0226^{*}$ \\
H2 & 0.7745 & 0.1891 & 0.7345 & $0.0264^{*}$ \\
H3 & 0.8174 & -0.0606 & 0.8659 & $0.0241^{*}$
\end{tabular}




\begin{tabular}{lllll}
$\mathrm{H} 4$ & 0.9138 & -0.2392 & 0.9391 & $0.0254^{*}$ \\
$\mathrm{H} 5$ & 0.7997 & 0.6051 & 0.4991 & $0.0260^{*}$ \\
\hline
\end{tabular}

Atomic displacement parameters $\left(\AA^{2}\right)$

\begin{tabular}{lllllll}
\hline & $U^{11}$ & $U^{22}$ & $U^{33}$ & $U^{12}$ & $U^{13}$ & $U^{23}$ \\
\hline Br1 & $0.0141(2)$ & $0.0192(3)$ & $0.0204(2)$ & $0.0019(2)$ & $0.00822(16)$ & $0.0016(2)$ \\
O1 & $0.0123(13)$ & $0.0238(16)$ & $0.0188(14)$ & $0.0028(13)$ & $0.0093(12)$ & $0.0037(14)$ \\
O2 & $0.0140(15)$ & $0.034(2)$ & $0.0266(17)$ & $0.0055(15)$ & $0.0090(13)$ & $0.0042(16)$ \\
O3 & $0.0218(16)$ & $0.039(3)$ & $0.0243(16)$ & $0.0025(16)$ & $0.0139(14)$ & $0.0031(16)$ \\
C1 & $0.0189(19)$ & $0.022(3)$ & $0.0196(18)$ & $0.002(3)$ & $0.0131(16)$ & $-0.000(3)$ \\
C2 & $0.019(2)$ & $0.020(3)$ & $0.017(2)$ & $0.0013(18)$ & $0.0104(18)$ & $-0.0010(17)$ \\
C3 & $0.021(2)$ & $0.017(3)$ & $0.022(2)$ & $-0.0013(16)$ & $0.0120(18)$ & $-0.0011(16)$ \\
C4 & $0.0107(19)$ & $0.027(3)$ & $0.027(3)$ & $-0.0006(19)$ & $0.0094(18)$ & $-0.003(2)$ \\
C5 & $0.018(2)$ & $0.024(3)$ & $0.023(2)$ & $-0.0074(19)$ & $0.0137(18)$ & $-0.0038(19)$ \\
C6 & $0.020(2)$ & $0.024(3)$ & $0.018(2)$ & $-0.0038(19)$ & $0.0097(18)$ & $-0.0027(18)$ \\
C7 & $0.0126(19)$ & $0.0132(19)$ & $0.022(2)$ & $-0.0012(18)$ & $0.0095(17)$ & $0.0004(18)$ \\
C8 & $0.0148(19)$ & $0.015(3)$ & $0.018(2)$ & $-0.0004(16)$ & $0.0071(17)$ & $-0.0004(15)$ \\
C9 & $0.0137(19)$ & $0.020(3)$ & $0.018(2)$ & $-0.0004(18)$ & $0.0102(17)$ & $-0.0031(18)$ \\
C10 & $0.024(3)$ & $0.021(3)$ & $0.020(2)$ & $0.0023(19)$ & $0.0115(18)$ & $0.0006(19)$ \\
& & & & & &
\end{tabular}

Geometric parameters $\left(\AA,{ }^{\circ}\right)$

\begin{tabular}{|c|c|c|c|}
\hline $\mathrm{Br} 1-\mathrm{C} 7$ & $1.892(4)$ & $\mathrm{C} 4-\mathrm{C} 8$ & $1.405(9)$ \\
\hline $\mathrm{O} 1-\mathrm{C} 1$ & $1.337(5)$ & $\mathrm{C} 5-\mathrm{C} 6$ & $1.393(6)$ \\
\hline $\mathrm{O} 1-\mathrm{C} 9$ & $1.381(7)$ & $\mathrm{C} 6-\mathrm{C} 7$ & $1.387(9)$ \\
\hline $\mathrm{O} 2-\mathrm{C} 3$ & $1.224(6)$ & $\mathrm{C} 7-\mathrm{C} 9$ & $1.387(6)$ \\
\hline $\mathrm{O} 3-\mathrm{C} 10$ & $1.205(8)$ & $\mathrm{C} 8-\mathrm{C} 9$ & $1.402(6)$ \\
\hline $\mathrm{C} 1-\mathrm{C} 2$ & $1.367(6)$ & $\mathrm{C} 1-\mathrm{H} 1$ & 0.950 \\
\hline $\mathrm{C} 2-\mathrm{C} 3$ & $1.475(9)$ & $\mathrm{C} 4-\mathrm{H} 2$ & 0.950 \\
\hline $\mathrm{C} 2-\mathrm{C} 10$ & $1.469(7)$ & $\mathrm{C} 5-\mathrm{H} 3$ & 0.950 \\
\hline $\mathrm{C} 3-\mathrm{C} 8$ & $1.469(7)$ & $\mathrm{C} 6-\mathrm{H} 4$ & 0.950 \\
\hline $\mathrm{C} 4-\mathrm{C} 5$ & $1.370(7)$ & $\mathrm{C} 10-\mathrm{H} 5$ & 0.950 \\
\hline $\mathrm{Br} 1 \cdots \mathrm{O} 1$ & $2.995(4)$ & $\mathrm{Br} 1 \cdots \mathrm{H} 1^{\mathrm{i}}$ & 2.9874 \\
\hline $\mathrm{O} 1 \cdots \mathrm{C} 3$ & $2.875(6)$ & $\mathrm{Br} 1 \cdots \mathrm{H} 1^{\mathrm{ii}}$ & 3.0464 \\
\hline $\mathrm{O} 2 \cdots \mathrm{C} 1$ & $3.581(7)$ & $\operatorname{Br} 1 \cdots H 4^{\text {xii }}$ & 3.2048 \\
\hline $\mathrm{O} 2 \cdots \mathrm{C} 4$ & $2.881(6)$ & $\operatorname{Br} 1 \cdots H 4^{\text {xiii }}$ & 3.1419 \\
\hline $\mathrm{O} 2 \cdots \mathrm{C} 10$ & $2.922(8)$ & $\mathrm{O} 1 \cdots \mathrm{H} 1^{\mathrm{ii}}$ & 3.0722 \\
\hline $\mathrm{O} 3 \cdots \mathrm{C} 1$ & $2.799(6)$ & $\mathrm{O} 2 \cdots \mathrm{H} 3^{\text {xiv }}$ & 2.7748 \\
\hline $\mathrm{C} 1 \cdots \mathrm{C} 7$ & $3.585(8)$ & $\mathrm{O} 2 \cdots \mathrm{H} 5^{\mathrm{v}}$ & 2.7432 \\
\hline $\mathrm{C} 1 \cdots \mathrm{C} 8$ & $2.760(9)$ & $\mathrm{O} 2 \cdots \mathrm{H} 5^{\mathrm{vi}}$ & 2.5376 \\
\hline $\mathrm{C} 2 \cdots \mathrm{C} 9$ & $2.787(7)$ & $\mathrm{O} 3 \cdots \mathrm{H} 1^{\text {iv }}$ & 3.5037 \\
\hline $\mathrm{C} 4 \cdots \mathrm{C} 7$ & $2.773(6)$ & $\mathrm{O} 3 \cdots \mathrm{H} 3^{\text {viii }}$ & 2.9507 \\
\hline $\mathrm{C} 5 \cdots \mathrm{C} 9$ & $2.775(9)$ & $\mathrm{O} 3 \cdots{ }^{3} 3^{\text {ix }}$ & 2.9772 \\
\hline $\mathrm{C} 6 \cdots \mathrm{C} 8$ & $2.796(7)$ & $\mathrm{O} 3 \cdots \mathrm{H} 4^{\text {viii }}$ & 2.5712 \\
\hline $\mathrm{Br} 1 \cdots \mathrm{O} 3^{\mathrm{i}}$ & $3.046(4)$ & $\mathrm{O} 3 \cdots \mathrm{H} 4^{\mathrm{ix}}$ & 3.4743 \\
\hline $\mathrm{O} 1 \cdots \mathrm{O} 1^{\mathrm{ii}}$ & $3.378(6)$ & $\mathrm{C} 2 \cdots \mathrm{H} 5^{\mathrm{iii}}$ & 3.4598 \\
\hline
\end{tabular}




\begin{tabular}{|c|c|c|c|}
\hline $\mathrm{O} 1 \cdots \mathrm{C} 1^{\mathrm{iii}}$ & $3.503(6)$ & $\mathrm{C} 3 \cdots \mathrm{H}^{\mathrm{v}}$ & 3.3207 \\
\hline $\mathrm{O} 1 \cdots \mathrm{C} 2^{\mathrm{iii}}$ & $3.562(6)$ & $\mathrm{C} 4 \cdots \mathrm{H} 2^{\mathrm{xv}}$ & 2.9781 \\
\hline $\mathrm{O} 2 \cdots \mathrm{C}^{\text {iv }}$ & $3.544(6)$ & $\mathrm{C} 4 \cdots \mathrm{H} 2^{\text {xiv }}$ & 3.3174 \\
\hline $\mathrm{O} 2 \cdots \mathrm{C} 10^{v}$ & $3.163(5)$ & $\mathrm{C} 4 \cdots \mathrm{H} 3^{\text {xiv }}$ & 3.3139 \\
\hline $\mathrm{O} 2 \cdots \mathrm{C} 10^{\mathrm{vi}}$ & $3.375(5)$ & $\mathrm{C} 5 \cdots \mathrm{H} 2^{\mathrm{iii}}$ & 3.5171 \\
\hline $\mathrm{O} 3 \cdots \mathrm{Br} 1^{\mathrm{vii}}$ & $3.046(4)$ & $\mathrm{C} 5 \cdots \mathrm{H} 2^{\mathrm{xv}}$ & 2.8601 \\
\hline $\mathrm{O} 3 \cdots \mathrm{C}^{\mathrm{viii}}$ & $3.452(6)$ & $\mathrm{C} 5 \cdots \mathrm{H} 4^{\mathrm{iv}}$ & 3.5832 \\
\hline $\mathrm{O} 3 \cdots \mathrm{C}^{\mathrm{ix}}$ & $3.347(6)$ & $\mathrm{C} 6 \cdots \mathrm{H} 4^{\mathrm{iv}}$ & 3.5732 \\
\hline $\mathrm{O} 3 \cdots \mathrm{C} 6^{\text {viii }}$ & $3.266(8)$ & $\mathrm{C} 10 \cdots \mathrm{H} 3^{\text {viii }}$ & 3.4200 \\
\hline $\mathrm{C} 1 \cdots \mathrm{O} 1^{\text {iv }}$ & $3.503(6)$ & $\mathrm{C} 10 \cdots \mathrm{H} 3^{\mathrm{ix}}$ & 3.5839 \\
\hline $\mathrm{C} 1 \cdots \mathrm{C} 10^{\mathrm{iii}}$ & $3.527(6)$ & $\mathrm{H} 1 \cdots \mathrm{Br} 1^{\mathrm{ii}}$ & 3.0464 \\
\hline $\mathrm{C} 2 \cdots \mathrm{O} 1^{\text {iv }}$ & $3.562(6)$ & $\mathrm{H} 1 \cdots \mathrm{Br} 1^{\mathrm{vii}}$ & 2.9874 \\
\hline $\mathrm{C} 2 \cdots \mathrm{C} 10^{\mathrm{iii}}$ & $3.497(7)$ & $\mathrm{H} 1 \cdots \mathrm{O} 1^{\mathrm{ii}}$ & 3.0722 \\
\hline $\mathrm{C} 3 \cdots \mathrm{C} 8^{\text {iv }}$ & $3.491(7)$ & $\mathrm{H} 1 \cdots \mathrm{O} 3^{\mathrm{iii}}$ & 3.5037 \\
\hline $\mathrm{C} 4 \cdots \mathrm{C} 5^{\text {iv }}$ & $3.511(7)$ & $\mathrm{H} 1 \cdots \mathrm{H} 4^{\mathrm{viii}}$ & 3.5860 \\
\hline$C 5 \cdots O 3^{x}$ & $3.452(6)$ & $\mathrm{H} 2 \cdots \mathrm{C}^{\mathrm{xv}}$ & 3.3174 \\
\hline $\mathrm{C} 5 \cdots \mathrm{O} 3^{\mathrm{xi}}$ & $3.347(6)$ & $\mathrm{H} 2 \cdots \mathrm{C}{ }^{\mathrm{xiv}}$ & 2.9781 \\
\hline $\mathrm{C} 5 \cdots \mathrm{C}^{4}{ }^{\mathrm{iii}}$ & $3.511(7)$ & $\mathrm{H} 2 \cdots \mathrm{C} 5^{\text {iv }}$ & 3.5171 \\
\hline $\mathrm{C} 6 \cdots 3^{\mathrm{x}}$ & $3.266(8)$ & $\mathrm{H} 2 \cdots \mathrm{C} 5^{\mathrm{xiv}}$ & 2.8601 \\
\hline $\mathrm{C} 7 \cdots \mathrm{C}^{\mathrm{iii}}$ & $3.592(6)$ & $\mathrm{H} 2 \cdots \mathrm{H} 2^{\mathrm{xv}}$ & 2.6156 \\
\hline $\mathrm{C} 7 \cdots{ }^{-} 9^{\mathrm{iii}}$ & $3.486(7)$ & $\mathrm{H} 2 \cdots \mathrm{H} 2^{\text {xiv }}$ & 2.6156 \\
\hline $\mathrm{C} 8 \cdots \mathrm{O} 2^{\mathrm{iii}}$ & $3.544(6)$ & $\mathrm{H} 2 \cdots \mathrm{H} 3^{\text {iv }}$ & 3.5746 \\
\hline $\mathrm{C} 8 \cdots \mathrm{C} 3^{\mathrm{iii}}$ & $3.491(7)$ & $\mathrm{H} 2 \cdots \mathrm{H} 3^{\text {xiv }}$ & 2.3921 \\
\hline $\mathrm{C} 8{ }^{\cdots} \mathrm{C} 7^{\mathrm{iv}}$ & $3.592(6)$ & $\mathrm{H} 3 \cdots \mathrm{O} 2^{\mathrm{xv}}$ & 2.7748 \\
\hline $\mathrm{C} 9 \cdots \mathrm{C}^{\mathrm{iv}}$ & $3.486(7)$ & $\mathrm{H} 3{ }^{\cdots} \mathrm{O} 3^{x}$ & 2.9507 \\
\hline $\mathrm{C} 10 \cdots \mathrm{O} 2^{v}$ & $3.163(5)$ & $\mathrm{H} 3{ }^{\cdots} \mathrm{O} 3^{\mathrm{xi}}$ & 2.9772 \\
\hline $\mathrm{C} 10 \cdots \mathrm{O} 2^{\mathrm{vi}}$ & $3.375(5)$ & $\mathrm{H} 3{ }^{\cdots} \mathrm{C} 4^{\mathrm{xv}}$ & 3.3139 \\
\hline $\mathrm{C} 10 \cdots \mathrm{C} 1^{\text {iv }}$ & $3.527(6)$ & $\mathrm{H} 3{ }^{\cdots} \mathrm{C} 10^{\mathrm{x}}$ & 3.4200 \\
\hline $\mathrm{C} 10 \cdots \mathrm{C} 2^{\mathrm{iv}}$ & $3.497(7)$ & $\mathrm{H} 3 \cdots \mathrm{C} 10^{\mathrm{xi}}$ & 3.5839 \\
\hline $\mathrm{Br} 1 \cdots \mathrm{H} 4$ & 2.9274 & $\mathrm{H} 3 \cdots \mathrm{H} 2^{\mathrm{iii}}$ & 3.5746 \\
\hline $\mathrm{O} 2 \cdots \mathrm{H} 2$ & 2.6153 & $\mathrm{H} 3{ }^{\cdots} \cdot \mathrm{H} 2^{\mathrm{xv}}$ & 2.3921 \\
\hline $\mathrm{O} 2 \cdots \mathrm{H} 5$ & 2.6505 & H3 $\cdots H 5^{x}$ & 3.5442 \\
\hline $\mathrm{O} 3 \cdots \mathrm{H} 1$ & 2.4620 & $\mathrm{H} 3 \cdots{ }^{\prime} 5^{\mathrm{xi}}$ & 3.3525 \\
\hline $\mathrm{C} 1 \cdots \mathrm{H} 5$ & 3.2749 & $\mathrm{H} 4 \cdots \mathrm{Br}^{\mathrm{xii}}$ & 3.2048 \\
\hline $\mathrm{C} 3 \cdots \mathrm{H} 1$ & 3.3068 & $\mathrm{H} 4 \cdots \mathrm{Br} 1^{\text {xiii }}$ & 3.1419 \\
\hline $\mathrm{C} 3 \cdots \mathrm{H} 2$ & 2.6753 & $\mathrm{H} 4 \cdots \mathrm{O}^{\mathrm{x}}$ & 2.5712 \\
\hline $\mathrm{C} 3 \cdots \mathrm{H} 5$ & 2.7288 & $\mathrm{H} 4 \cdots{ }^{\cdots} 3^{\mathrm{xi}}$ & 3.4743 \\
\hline $\mathrm{C} 4 \cdots \mathrm{H} 4$ & 3.2489 & $\mathrm{H} 4 \cdots \mathrm{C} 5^{\mathrm{iii}}$ & 3.5832 \\
\hline $\mathrm{C} 6 \cdots \mathrm{H} 2$ & 3.2487 & $\mathrm{H} 4 \cdots \mathrm{C}^{\mathrm{iii}}$ & 3.5732 \\
\hline $\mathrm{C} 7 \cdots \mathrm{H} 3$ & 3.2654 & $\mathrm{H} 4{ }^{\cdots} \cdot \mathrm{H} 1^{\mathrm{x}}$ & 3.5860 \\
\hline $\mathrm{C} 8 \cdots \mathrm{H} 3$ & 3.2750 & $\mathrm{H} 5 \cdots \mathrm{O} 2^{\mathrm{v}}$ & 2.7432 \\
\hline $\mathrm{C} 9 \cdots \mathrm{H} 1$ & 3.1865 & $\mathrm{H} 5 \cdots \mathrm{O} 2^{\mathrm{vi}}$ & 2.5376 \\
\hline $\mathrm{C} 9 \cdots \mathrm{H} 2$ & 3.2660 & $\mathrm{H} 5 \cdots \mathrm{C} 2^{\text {iv }}$ & 3.4598 \\
\hline C9 $\cdots \mathrm{H} 4$ & 3.2581 & $\mathrm{H} 5 \cdots \mathrm{C} 3^{\mathrm{v}}$ & 3.3207 \\
\hline $\mathrm{C} 10 \cdots \mathrm{H} 1$ & 2.5371 & $\mathrm{H} 5 \cdots 3^{\text {viii }}$ & 3.5442 \\
\hline $\mathrm{H} 1 \cdots \mathrm{H} 5$ & 3.4755 & $\mathrm{H} 5 \cdots \mathrm{H} 3^{\text {ix }}$ & 3.3525 \\
\hline $\mathrm{H} 2 \cdots \mathrm{H} 3$ & 2.3112 & $\mathrm{H} 5 \cdots \mathrm{H} 5^{\mathrm{vi}}$ & 3.0057 \\
\hline $\mathrm{H} 3 \cdots \mathrm{H} 4$ & 2.3430 & & \\
\hline
\end{tabular}




\begin{tabular}{|c|c|c|c|}
\hline $\mathrm{C} 1-\mathrm{O} 1-\mathrm{C} 9$ & $118.5(4)$ & $\mathrm{C} 4-\mathrm{C} 8-\mathrm{C} 9$ & $118.0(4)$ \\
\hline $\mathrm{O} 1-\mathrm{C} 1-\mathrm{C} 2$ & $125.2(6)$ & $\mathrm{O} 1-\mathrm{C} 9-\mathrm{C} 7$ & $117.3(4)$ \\
\hline $\mathrm{C} 1-\mathrm{C} 2-\mathrm{C} 3$ & $119.7(5)$ & $\mathrm{O} 1-\mathrm{C} 9-\mathrm{C} 8$ & $121.9(4)$ \\
\hline $\mathrm{C} 1-\mathrm{C} 2-\mathrm{C} 10$ & $118.2(6)$ & $\mathrm{C} 7-\mathrm{C} 9-\mathrm{C} 8$ & $120.8(5)$ \\
\hline $\mathrm{C} 3-\mathrm{C} 2-\mathrm{C} 10$ & $122.1(4)$ & $\mathrm{O} 3-\mathrm{C} 10-\mathrm{C} 2$ & $124.2(4)$ \\
\hline $\mathrm{O} 2-\mathrm{C} 3-\mathrm{C} 2$ & $122.5(5)$ & $\mathrm{O} 1-\mathrm{C} 1-\mathrm{H} 1$ & 117.386 \\
\hline $\mathrm{O} 2-\mathrm{C} 3-\mathrm{C} 8$ & $123.2(6)$ & $\mathrm{C} 2-\mathrm{C} 1-\mathrm{H} 1$ & 117.377 \\
\hline $\mathrm{C} 2-\mathrm{C} 3-\mathrm{C} 8$ & $114.3(4)$ & $\mathrm{C} 5-\mathrm{C} 4-\mathrm{H} 2$ & 119.373 \\
\hline $\mathrm{C} 5-\mathrm{C} 4-\mathrm{C} 8$ & $121.3(4)$ & $\mathrm{C} 8-\mathrm{C} 4-\mathrm{H} 2$ & 119.362 \\
\hline $\mathrm{C} 4-\mathrm{C} 5-\mathrm{C} 6$ & $120.0(6)$ & $\mathrm{C} 4-\mathrm{C} 5-\mathrm{H} 3$ & 120.002 \\
\hline $\mathrm{C} 5-\mathrm{C} 6-\mathrm{C} 7$ & $120.1(5)$ & $\mathrm{C} 6-\mathrm{C} 5-\mathrm{H} 3$ & 120.002 \\
\hline $\mathrm{Br} 1-\mathrm{C} 7-\mathrm{C} 6$ & $120.2(3)$ & $\mathrm{C} 5-\mathrm{C} 6-\mathrm{H} 4$ & 119.976 \\
\hline $\mathrm{Br} 1-\mathrm{C} 7-\mathrm{C} 9$ & $120.0(4)$ & $\mathrm{C} 7-\mathrm{C} 6-\mathrm{H} 4$ & 119.964 \\
\hline $\mathrm{C} 6-\mathrm{C} 7-\mathrm{C} 9$ & $119.8(4)$ & $\mathrm{O} 3-\mathrm{C} 10-\mathrm{H} 5$ & 117.902 \\
\hline $\mathrm{C} 3-\mathrm{C} 8-\mathrm{C} 4$ & $121.6(4)$ & $\mathrm{C} 2-\mathrm{C} 10-\mathrm{H} 5$ & 117.903 \\
\hline $\mathrm{C} 3-\mathrm{C} 8-\mathrm{C} 9$ & $120.4(6)$ & & \\
\hline $\mathrm{C} 1-\mathrm{O} 1-\mathrm{C} 9-\mathrm{C} 7$ & $179.6(4)$ & $\mathrm{C} 8-\mathrm{C} 4-\mathrm{C} 5-\mathrm{C} 6$ & $-0.2(7)$ \\
\hline $\mathrm{C} 1-\mathrm{O} 1-\mathrm{C} 9-\mathrm{C} 8$ & $0.7(6)$ & $\mathrm{C} 8-\mathrm{C} 4-\mathrm{C} 5-\mathrm{H} 3$ & 179.8 \\
\hline $\mathrm{C} 9-\mathrm{O} 1-\mathrm{C} 1-\mathrm{C} 2$ & $0.1(6)$ & $\mathrm{H} 2-\mathrm{C} 4-\mathrm{C} 5-\mathrm{C} 6$ & 179.8 \\
\hline $\mathrm{C} 9-\mathrm{O} 1-\mathrm{C} 1-\mathrm{H} 1$ & -179.9 & $\mathrm{H} 2-\mathrm{C} 4-\mathrm{C} 5-\mathrm{H} 3$ & -0.2 \\
\hline $\mathrm{O} 1-\mathrm{C} 1-\mathrm{C} 2-\mathrm{C} 3$ & $-0.9(7)$ & $\mathrm{H} 2-\mathrm{C} 4-\mathrm{C} 8-\mathrm{C} 3$ & 0.7 \\
\hline $\mathrm{O} 1-\mathrm{C} 1-\mathrm{C} 2-\mathrm{C} 10$ & $178.9(4)$ & $\mathrm{H} 2-\mathrm{C} 4-\mathrm{C} 8-\mathrm{C} 9$ & 179.8 \\
\hline $\mathrm{H} 1-\mathrm{C} 1-\mathrm{C} 2-\mathrm{C} 3$ & 179.1 & $\mathrm{C} 4-\mathrm{C} 5-\mathrm{C} 6-\mathrm{C} 7$ & $-0.6(7)$ \\
\hline $\mathrm{H} 1-\mathrm{C} 1-\mathrm{C} 2-\mathrm{C} 10$ & -1.1 & $\mathrm{C} 4-\mathrm{C} 5-\mathrm{C} 6-\mathrm{H} 4$ & 179.4 \\
\hline $\mathrm{C} 1-\mathrm{C} 2-\mathrm{C} 3-\mathrm{O} 2$ & $179.6(4)$ & $\mathrm{H} 3-\mathrm{C} 5-\mathrm{C} 6-\mathrm{C} 7$ & 179.4 \\
\hline $\mathrm{C} 1-\mathrm{C} 2-\mathrm{C} 3-\mathrm{C} 8$ & $0.7(6)$ & $\mathrm{H} 3-\mathrm{C} 5-\mathrm{C} 6-\mathrm{H} 4$ & -0.6 \\
\hline $\mathrm{C} 1-\mathrm{C} 2-\mathrm{C} 10-\mathrm{O} 3$ & $0.1(7)$ & $\mathrm{C} 5-\mathrm{C} 6-\mathrm{C} 7-\mathrm{Br} 1$ & $-179.6(4)$ \\
\hline $\mathrm{C} 1-\mathrm{C} 2-\mathrm{C} 10-\mathrm{H} 5$ & -179.9 & $\mathrm{C} 5-\mathrm{C} 6-\mathrm{C} 7-\mathrm{C} 9$ & $1.8(7)$ \\
\hline $\mathrm{C} 3-\mathrm{C} 2-\mathrm{C} 10-\mathrm{O} 3$ & $179.8(4)$ & $\mathrm{H} 4-\mathrm{C} 6-\mathrm{C} 7-\mathrm{Br} 1$ & 0.4 \\
\hline $\mathrm{C} 3-\mathrm{C} 2-\mathrm{C} 10-\mathrm{H} 5$ & -0.2 & $\mathrm{H} 4-\mathrm{C} 6-\mathrm{C} 7-\mathrm{C} 9$ & -178.2 \\
\hline $\mathrm{C} 10-\mathrm{C} 2-\mathrm{C} 3-\mathrm{O} 2$ & $-0.1(7)$ & $\mathrm{Br} 1-\mathrm{C} 7-\mathrm{C} 9-\mathrm{O} 1$ & $0.4(6)$ \\
\hline $\mathrm{C} 10-\mathrm{C} 2-\mathrm{C} 3-\mathrm{C} 8$ & $-179.0(4)$ & $\mathrm{Br} 1-\mathrm{C} 7-\mathrm{C} 9-\mathrm{C} 8$ & $179.2(3)$ \\
\hline $\mathrm{O} 2-\mathrm{C} 3-\mathrm{C} 8-\mathrm{C} 4$ & $0.3(6)$ & $\mathrm{C} 6-\mathrm{C} 7-\mathrm{C} 9-\mathrm{O} 1$ & $179.1(4)$ \\
\hline $\mathrm{O} 2-\mathrm{C} 3-\mathrm{C} 8-\mathrm{C} 9$ & $-178.8(4)$ & $\mathrm{C} 6-\mathrm{C} 7-\mathrm{C} 9-\mathrm{C} 8$ & $-2.1(7)$ \\
\hline $\mathrm{C} 2-\mathrm{C} 3-\mathrm{C} 8-\mathrm{C} 4$ & $179.1(4)$ & $\mathrm{C} 3-\mathrm{C} 8-\mathrm{C} 9-\mathrm{O} 1$ & $-0.8(6)$ \\
\hline $\mathrm{C} 2-\mathrm{C} 3-\mathrm{C} 8-\mathrm{C} 9$ & $0.1(6)$ & $\mathrm{C} 3-\mathrm{C} 8-\mathrm{C} 9-\mathrm{C} 7$ & $-179.6(4)$ \\
\hline $\mathrm{C} 5-\mathrm{C} 4-\mathrm{C} 8-\mathrm{C} 3$ & $-179.3(4)$ & $\mathrm{C} 4-\mathrm{C} 8-\mathrm{C} 9-\mathrm{O} 1$ & $-179.9(4)$ \\
\hline $\mathrm{C} 5-\mathrm{C} 4-\mathrm{C} 8-\mathrm{C} 9$ & $-0.2(7)$ & $\mathrm{C} 4-\mathrm{C} 8-\mathrm{C} 9-\mathrm{C} 7$ & $1.3(6)$ \\
\hline
\end{tabular}

Symmetry codes: (i) $-x+2, y-1,-z+3 / 2$; (ii) $-x+2, y,-z+3 / 2$; (iii) $x, y-1, z$; (iv) $x, y+1, z$; (v) $-x+3 / 2,-y+1 / 2,-z+1$; (vi) $-x+3 / 2,-y+3 / 2,-z+1$; (vii) $-x+2$, $y+1,-z+3 / 2$; (viii) $x,-y, z-1 / 2$; (ix) $x,-y+1, z-1 / 2$; (x) $x,-y, z+1 / 2$; (xi) $x,-y+1, z+1 / 2$; (xii) $-x+2,-y-1,-z+2$; (xiii) $-x+2,-y,-z+2$; (xiv) $-x+3 / 2$, $y+1 / 2,-z+3 / 2 ;(\mathrm{xv})-x+3 / 2, y-1 / 2,-z+3 / 2$.

Hydrogen-bond geometry $\left(A,{ }^{\circ}\right)$

\begin{tabular}{lllll}
\hline$D-\mathrm{H} \cdots A$ & $D-\mathrm{H}$ & $\mathrm{H} \cdots A$ & $D \cdots A$ & $D-\mathrm{H} \cdots A$ \\
\hline $\mathrm{C} 10-\mathrm{H} 5 \cdots \mathrm{O} 2^{\mathrm{vi}}$ & 0.95 & 2.54 & $3.375(5)$ & $147(1)$
\end{tabular}


$\mathrm{C} 7-\mathrm{Br} 1 \cdots \mathrm{O} 3^{\mathrm{i}}$

$\mathrm{C} 10-\mathrm{O} 3 \cdots \mathrm{Br}^{\mathrm{vii}}$

Symmetry codes: (i) $-x+2, y-1,-z+3 / 2$; (vi) $-x+3 / 2,-y+3 / 2,-z+1$; (vii) $-x+2, y+1,-z+3 / 2$.
$4.934(6)$

$3.962(6)$
$175(1)$

$133(1)$

$\begin{array}{llll}1.89(1) & 3.05(1) & 4.934(6) & 175(1) \\ 1.21(1) & 3.05(1) & 3.962(6) & 133(1)\end{array}$

